05

\title{
Электрические и магнитные свойства диселенида ванадия, интеркалированного атомами хрома
}

\author{
(C) Е.М. Шерокалова, Н.В. Селезнева, В.Г. Плещев \\ Институт естественных наук и математики Уральского федерального университета им. Б.Н. Ельцина, \\ Екатеринбург, Россия \\ E-mail: v.g.pleshchev@urfu.ru
}

Поступила в Редакцию 17 декабря 2021 г.

В окончательной редакции 17 декабря 2021 г.

Принята к публикации 27 декабря 2021 г.

\begin{abstract}
Исследование структурных характеристик, электрических и магнитных свойств выполнено на синтезированных образцах диселенида ванадия, интеркалированного атомами хрома. Структурные исследования показали понижение симметрии кристаллической решетки от гексагональной до моноклинной при возрастании концентрации интеркалированных атомов, вызванное их упорядочением в Ван-дер-Ваальсовой щели. При этом происходит изменение резистивного состояния в образцах разного состава, которые характеризуется противоположными термическими коэффициентами электросопротивления. Значения эффективных магнитных моментов ионов хрома в $\mathrm{Cr}_{x} \mathrm{VSe}_{2}$ уменьшаются по сравнению со спиновыми характеристиками по мере увеличения содержания хрома и их изменение коррелирует с концентрационной зависимостью параметра с элементарной ячейки. При низких температурах для составов $x>0.2$ происходит переход соединений в состояние спинового стекла с критической температурой до $30 \mathrm{~K}$.
\end{abstract}

Ключевые слова: диселенид ванадия, хром, интеркалирование электросопротивление, магнитная восприимчивость, спиновое стекло.

DOI: $10.21883 /$ FTT.2022.04.52183.256

\section{1. Введение}

Слоистые дихалькогениды переходных металлов (СДПМ) с общей формулой $T X_{2}$, где $T-$ переходный метал IV, V групп, $X$ - халькоген) имеют ярко выраженный квазидвумерный характер кристаллической структуры, что является следствием наличия так называемой „Ван-дер-Ваальсовой щели“ (ВдВ) между трехслойными блоками („,ээндичами“) $X-T-X$, куда оказывается возможным внедрять (интеркалировать) атомы различных элементов или даже целые молекулы [1-2].

При внедрении инородных атомов в СДПМ основной мотив кристаллической структуры, как правило, сохраняется. Тем не менее, различным концентрациям интеркалированных атомов в этих соединениях соответствуют различные структурные модификации, вызванные различным количеством слоев $T X_{2}$ в элементарной ячейке, а также с упорядочением вакансий и внедренных атомов в ВдВ щелях. Упорядочение, происходящее в подрешетке интеркаланта, кроме увеличения объема элементарной ячейки, сопровождается понижением ее симметрии [3-5]. Как показали ранее проведенные исследования, физические свойства соединений, получаемых путем интеркалирования атомами $3 d$-переходных элементов, существенно отличаются от свойств исходных соединений $T X_{2}$. Дополнительные взаимодействия внедренных атомов с атомами матрицы приводят к деформации кристаллической решетки, изменению электрических и магнитных свойств интекалированных материалов, к возникновению различных магнитных состояний $[6,7]$.

Среди подобных соединений наиболее изученными являются дихалькогениды титана и интеркалаты на их основе, кристаллическая структура которых представляется одним $T X_{2}$ слоем на элементарную ячейку. Такую же структуру имеют некоторые дихалькогениды элементов 5 группы и, в частности, диселенид ванадия. $\mathrm{B}$ этом соединении, как и в $\mathrm{TiSe}_{2}$, наблюдается переход в состояние с волной зарядовой плотности, хотя и при более низких температурах (110 К) $[3,8]$.

Интеркалирование $\mathrm{VSe}_{2}$ атомами других элементов, как и в соединениях на основе диселенида титана, а также процессы самоинтеркалации, приводящие к возникновению искажений кристаллической структуры, подавляют это структурное превращение. Так, например, самоинтеркалация в диселениде ванадия $\mathrm{V}_{x} \mathrm{VSe}_{2}$ приводит к подавлению состояния с ВЗП уже при $x>0.01$, а в соединении $\mathrm{Fe}_{x} \mathrm{VSe}_{2}$ при $x>0.033[9,10]$.

Несмотря на подобие кристаллических структур различных СДПМ, при анализе механизмов формирования физических свойств интеркалированных соединений необходимо принимать во внимание не только различия в природе и электронном строении внедряемых атомов $3 d$-элементов, но и характерные особенности исходных дихалькогенидов, используемых для интеркалирования. Так, например, в системе $\mathrm{Cr}_{x} \mathrm{TiSe}_{2}$ при $x=0.33$ обнаружено антиферромагнитное состояние, а в $\mathrm{Cr}_{x} \mathrm{TiTe}_{2}$ и $\mathrm{Cr}_{x} \mathrm{MoSe}_{2}$ - формируется ферромагнитное упорядочение $[11,12]$. 
В связи с этим, настоящая работа посвящена комплексному исследованию структурных особенностей, электрических и магнитных свойств диселенида ванадия при его интеркалировании атомами хрома.

\section{2. Эксперимент}

Поликристаллические образцы $\mathrm{Cr}_{x} \mathrm{VSe}_{2}$ были получены методом твердофазного синтеза в вакуумированных кварцевых ампулах в две стадии. На первой стадии синтезировались образцы бинарного соединения $\mathrm{VSe}_{2}$, которые впоследствии служили матрицами для интеркалирования. На второй стадии готовились образцы интеркалированных соединений путем смешивания полученных матриц с необходимым количеством хрома согласно реакции $x \mathrm{Cr}+\mathrm{VSe}_{2}=\mathrm{Cr}_{x} \mathrm{VSe}_{2}(0 \leq x \leq 0.33)$. Температура синтеза на каждом этапе составляла $800^{\circ} \mathrm{C}$ и длительность выдержки не менее $100 \mathrm{~h}$. На каждой стадии после первичного отжига производилось перетирание и прессование полученных препаратов и повторный гомогенизационный отжиг. В случае необходимости подобные операции проводились повторно. Подобная методика хорошо зарекомендовала себя при синтезе других интеркалированных систем [5-7]. Достоинством ее является более вероятное предотвращение образования бинарных халькогенидов обоих металлов в случае непосредственного синтеза из исходных элементов. Контроль фазового состава и определение структурных характеристик были выполнены с использованием рентгеновского дифрактометра Bruker D8 Advance и метода полнопрофильного анализа рентгенографических данных.

Измерения электросопротивления проводились стандартным четырехзондовым методом в интервале температур 100-300 K. Намагниченность измерялась на СКВИД-магнитометре (MPMS) в интервале температур $2-300 \mathrm{~K}$ и диапазоне магнитных полей до $60 \mathrm{kOe}$.

В результате рентгенографической аттестации была подтверждена однофазность полученных образцов, определены параметры элементарных ячеек. Было установлено, что рентгенограммы образцов $\mathrm{Cr}_{x} \mathrm{VSe}_{2}$ с малым содержанием хрома $(0.05 \leq x \leq 0.2)$ индицируются в рамках тригональной сингонии с пространственной группой $P \overline{3} m 1$, соответствующей исходному $\mathrm{VSe}_{2}$. При увеличении содержания хрома в образцах $\mathrm{Cr}_{0.25} \mathrm{VSe}_{2}$ и $\mathrm{Cr}_{0.33} \mathrm{VSe}_{2}$ было обнаружено упорядочение атомов хрома. Их рентгенограммы индицировались в моноклинной сингонии $(\mathrm{SG}=I 2 / m)$ с параметрами элементарной ячейки $2 \sqrt{ } 3 a_{0} \times 2 a_{0} \times 2 c_{0}$, где $a_{0}$ и $c_{0}$ параметры элементарной ячейки исходного диселенида ванадия.

\section{3. Результаты}

При измерениях электросопротивления было обнаружено, что температурные зависимости образцов с различным содержанием хрома имеют разный характер.
Для $\mathrm{VSe}_{2}$ зависимость $\rho(T)$ является строго линейной, что позволяет сделать вывод о преимущественно электрон-фононном механизме рассеяния носителей заряда. При внедрении хрома $(x=0.05$ и $x=0.1)$ величина электросопротивления возрастает. Величина $\rho$ также увеличивается с ростом температуры, но зависимость $\rho(T)$ перестает быть линейной и свидетельствует о том, что помимо фононного рассеяния возникает дополнительный механизм. Эти зависимости в большей степени соответствует виду $\rho(T)=\rho_{0}+a T^{m}$, где $m>1$. Определенные нами путем аппроксимации величины т для этих двух образцов составляют соответственно 1.4 и 1.7 , что позволяет предполагать, что природа дополнительного вклада в электросопротивление может быть связана с электрон-электронным рассеянием, поскольку данный механизм, согласно имеющимся представлениям, может приводить к зависимости $\rho(T) \infty T^{2}[13]$. Величина температурно-независимого члена $\rho_{0}$ в уравнении для $\rho(T)$ должна представлять собой сопротивление, вызванное наличием собственных структурных дефектов, а также примесей, роль которых играют внедренные атомы (ионы) хрома (рис. 1). При оценке этого вклада в электросопротивление были получены значения равные $1.05 \cdot 10^{-5}$ и $1.93 \cdot 10^{-5} \Omega \cdot \mathrm{m}$. Практически двукратное увеличение $\rho_{0}$ соответствует такому же возрастанию содержания хрома в образцах с $x=0.05$ и $x=0.1$. При увеличении содержания хрома при $x \geq 0.2$ зависимость $\rho(T)$ претерпевает качественные изменения и характеризуется отрицательным температурным коэффициентом. Если рассматривать такую зависимость как проявление активационного процесса, то сделанные нами оценки энергии активации для этих образцов показали значения от 2 до $5 \mathrm{meV}$, что определяет довольно слабое изменение $\rho$ в данном интервале температур. Переходный случай наблюдается для состава $\mathrm{Cr}_{0.15} \mathrm{VSe}_{2}$, где по мере повышения температуры происходит смена знака температурного коэффициента электросопротивления (рис. 2). На рис. 2 представлена также сводная концентрационная зависимость электросопротивления образцов $\mathrm{Cr}_{x} \mathrm{VSe}_{2}$ при двух температурах. Заштрихованная область, содержащая состав с $x=0.15$, представляет собой вероятную область содержаний атомов хрома, в пределах которой происходит изменение характера температурной зависимости электросопротивления. Наблюдаемое изменение резистивного состояния может быть вызвано отмеченными выше процессами упорядочения интеркалированных атомов и связанными с этим изменениями в электронной структуре соединений [14].

При увеличении содержания хрома от $x=0.2$ до $x=0.33$ величина $\rho$ испытывает рост, как это происходит и для меньших концентраций $(x=0.05$ и $x=0.1)$. Таким образом, следует заключить, что несмотря на возможное проявление активационного процесса в образцах со структурным упорядочением, общая концентрационная зависимость электросопротивления определяется увеличивающимся рассеянием носителей заряда при росте содержания хрома в $\mathrm{Cr}_{x} \mathrm{VSe}_{2}$. 

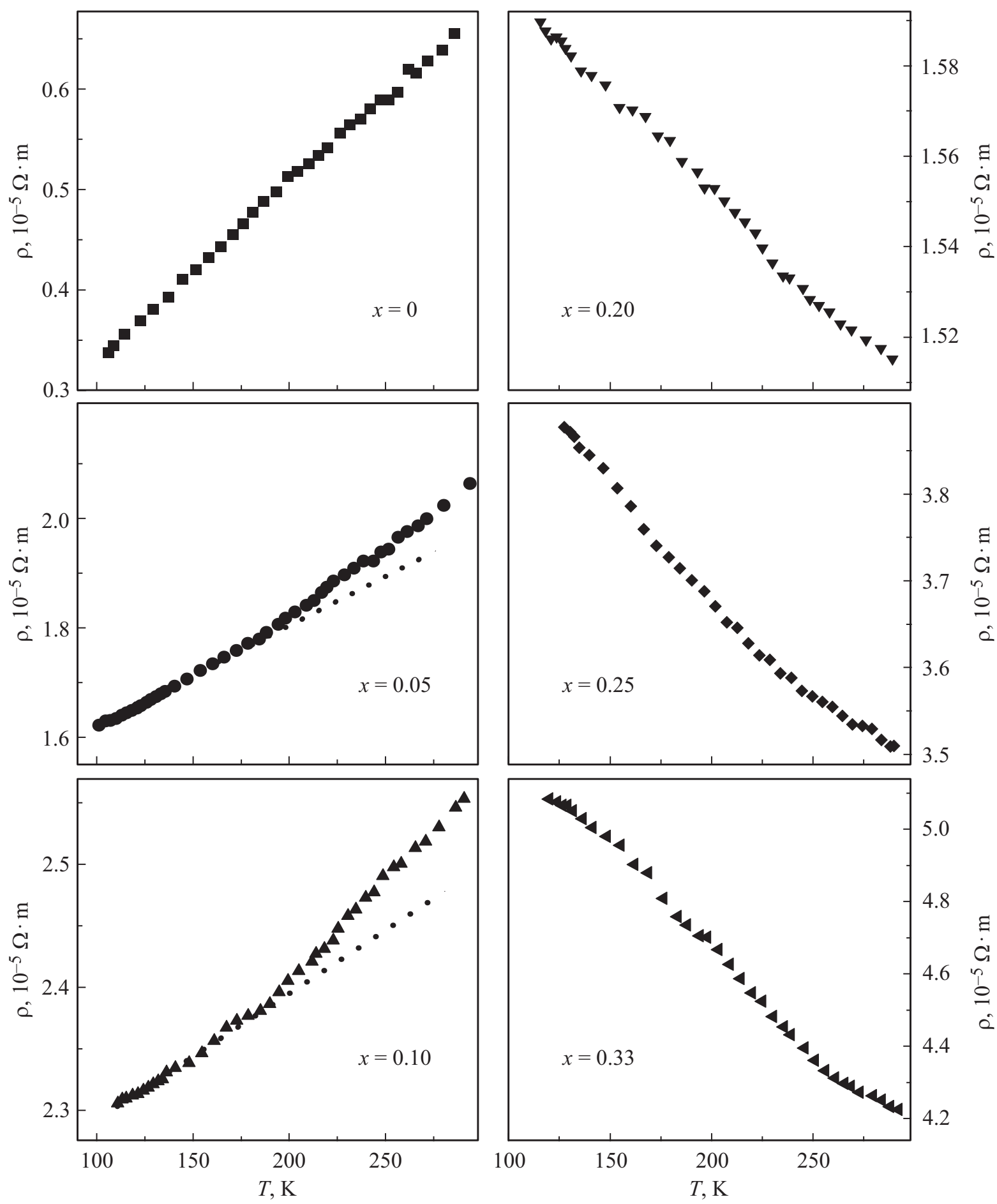

Рис. 1. Температурные зависимости электросопротивления образцов $\mathrm{Cr}_{x} \mathrm{VSe}_{2}$.

Температурные зависимости магнитной восприимчивости $(\chi) \mathrm{Cr}_{x} \mathrm{VSe}_{2}$, измеренные в магнитном поле $H=10 \mathrm{kOe}$, представлены на рис. 3. Эти зависимости у образцов с $x=0,0.05,0.1$ во всей температурной области демонстрируют монотонное уменьшение восприимчивости при нагревании и ее увеличение при росте $x$. У образцов с $x=0.2,0.25,0.33$ в области низких температур монотонные зависимости $\chi(T)$ нарушаются, а величина магнитной восприимчивости при росте $x$ уменьшается. В интервале температур $70-300 \mathrm{~K}$ температурные зависимости магнитной восприимчивости интеркалированных соединений были обработаны в соответствии с законом Кюри-Вейсса в виде $\chi=\chi_{0}+C /(T-\Theta)$, где $C-$ постоянная Кюри, $\Theta-$ температура Кюри-Вейсса, $\chi_{0}-$ температурнонезависимый вклад, включающий в себя диамагнетизм 
Значения температурно-независимого вклада $\chi_{0}$, температуры Кюри-Вейсса $\Theta$, эффективных магнитных моментов формульной единицы соединений $\mathrm{Cr}_{x} \mathrm{VSe}_{2} \mu_{\text {eff }} / \mathrm{f}$.u. и ионов хрома $\mu_{\text {eff }} / \mathrm{Cr}$ в зависимости от содержания хрома

\begin{tabular}{c|c|c|c|c}
\hline$x$ & $\begin{array}{c}\chi_{0}, 10^{-6} \\
\mathrm{emu} /(\mathrm{g} \cdot \mathrm{Oe})\end{array}$ & $\Theta, \mathrm{K}$ & $\mu_{\text {eff }, / f . u .,} \mu_{\mathrm{B}}$ & $\mu_{\mathrm{eff}} / \mathrm{Cr}, \mu_{\mathrm{B}}$ \\
\hline 0 & 1.3 & -0.5 & 0.46 & 0 \\
0.05 & 1.4 & -0.8 & 0.85 & 3.78 \\
0.1 & 1.6 & -6.2 & 1.20 & 3.75 \\
0.2 & 2.4 & -10 & 1.51 & 3.39 \\
0.25 & 4.4 & -24 & 1.45 & 2.86 \\
0.33 & 3.1 & -16 & 1.55 & 2.67
\end{tabular}

заполненных электронных оболочек и парамагнетизм электронного газа. В таблице приведены величины этих параметров, полученных в результате аппроксимации.

Поскольку в данных соединениях ион $\mathrm{V}^{4+}$, как и ион хрома, может обладать нескомпенсированным магнитным моментом, то определение эффективных магнитных моментов из экспериментальных данных было выполнено в расчете на формульную единицу этих соединений.

Как видно, интеркалация хромом ведет к увеличению $\mu_{\text {eff }} /$ f.u., определенного из эксперимента и рассчитанного на формульную единицу соединения.

Поскольку величина этих моментов определяется одновременно вкладом самой матрицы и интеркалированными ионами хрома, то экспериментальная величина $\mu_{\text {eff }} /$.u. может быть представлена в виде уравнения, из которого были определены эффективные магнитные моменты ионов хрома в соединениях $\mathrm{Cr}_{x} \mathrm{VSe}_{2}$

$$
\left.\left(\mu_{\mathrm{eff}}^{\exp }\right)^{2}=\left(\mu_{\mathrm{eff}}^{\mathrm{VSe}_{2}}\right)^{2}+x \cdot \mu_{\mathrm{eff}}^{\mathrm{Cr}}\right)^{2}
$$

где для $\mu_{\text {eff }}^{\mathrm{VSe}_{2}}$ принято значение, полученное для исходной матрицы. Определенные таким способом эффек-

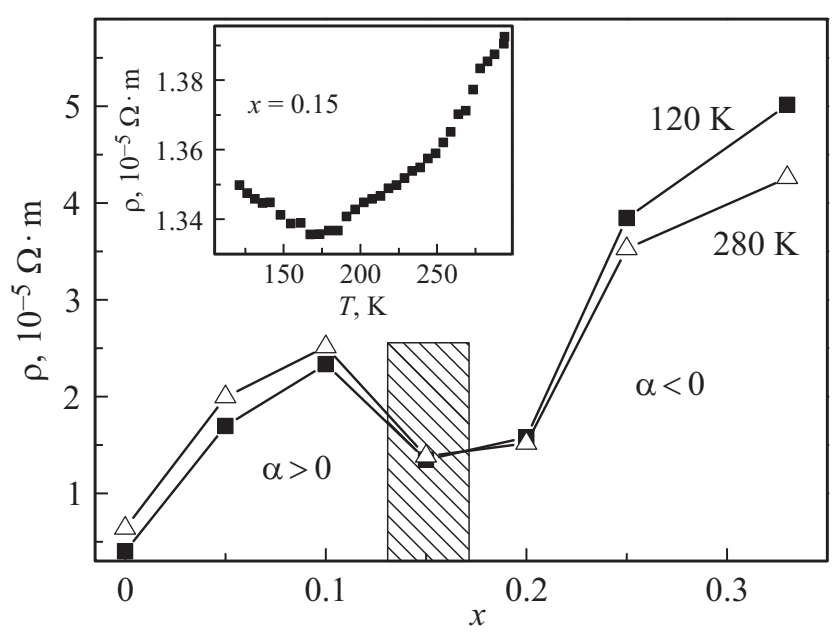

Рис. 2. Концентрационные зависимости электросопротивления в системе $\mathrm{Cr}_{x} \mathrm{VSe}_{2}$ и температурная зависимость электросопротивления $\mathrm{Cr}_{0.15} \mathrm{VSe}_{2}$ (на вставке).

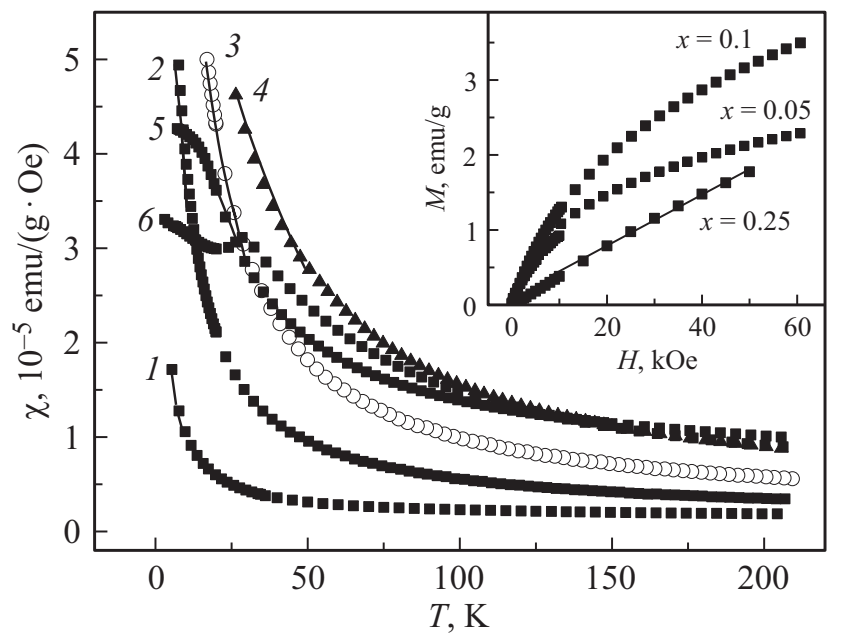

Рис. 3. Температурные зависимости магнитной восприимчивости образцов $\mathrm{Cr}_{x} \mathrm{VSe}_{2}$ при $x=0(1), x=0.05$ (2), $x=0.1$ (3), $x=0.2(4), x=0.25(5), x=0.33(6)$. На вставке - полевые зависимости намагниченности соединений $\mathrm{Cr}_{x} \mathrm{VSe}_{2}$ при $T=2 \mathrm{~K}$.

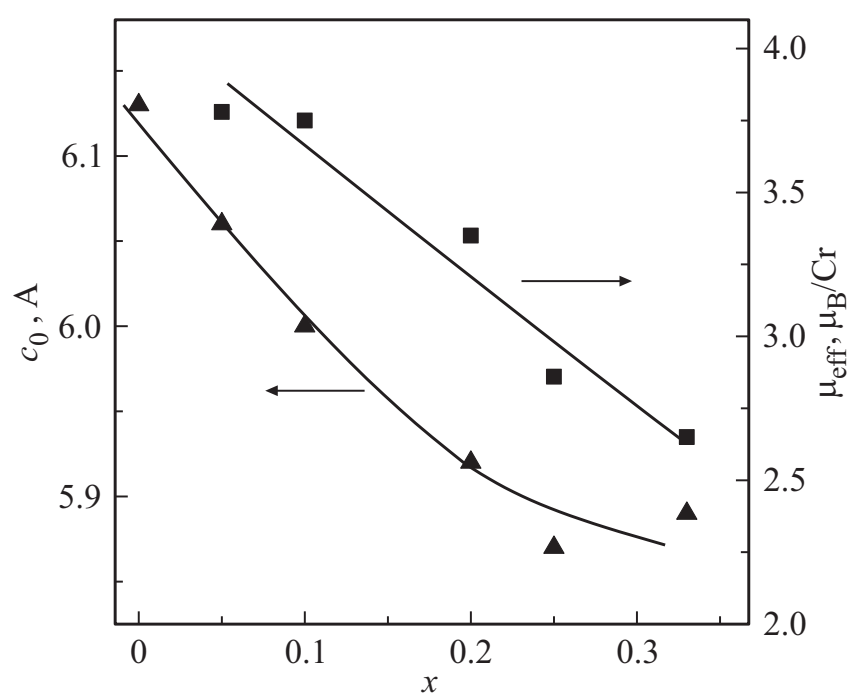

Рис. 4. Концентрационные зависимости параметра элементарной ячейки $c_{0}$ и эффективного магнитного момента на ион хрома в $\mathrm{Cr}_{x} \mathrm{VSe}_{2}$.

тивные магнитные моменты на ион хрома также представлены в таблице. То, что эти значения уменьшаются по сравнению со спиновым моментом для свободного иона $\mathrm{Cr}^{3+}\left(3.87 \mu_{\mathrm{B}}\right)$ свидетельствует, что $3 d$-электроны хрома испытывают заметную гибридизацию с состояниями $\mathrm{VSe}_{2}$. Эта гибридизация обуславливает также уменьшение параметра элементарной ячейки $c$ в направлении, перпендикулярном плоскости слоев, по мере роста содержания хрома, которое было нами обнаружено в результате рентгенографических исследований. На рис. 4 показана корреляция между изменениями $\mu_{\mathrm{eff}} / \mathrm{Cr}$ и параметра $c_{0}$. 


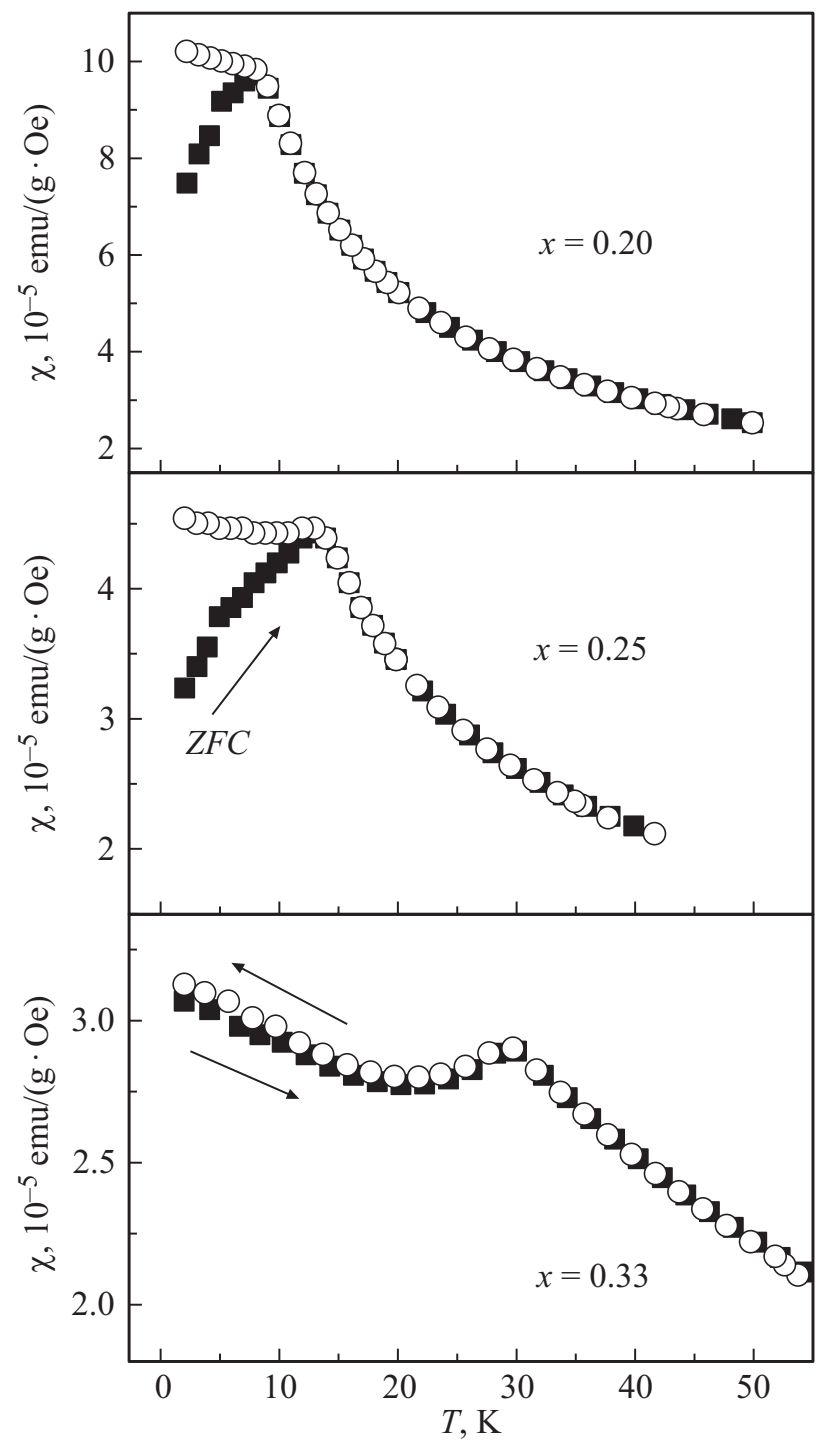

Рис. 5. Температурные зависимости магнитной восприимчивости $\mathrm{Cr}_{x} \mathrm{VSe}_{2}$ в режимах ZFC и FC $(H=100 \mathrm{Oe})$.

Магнитная восприимчивость в области низких температур была дополнительно исследована в режимах ZFC и $\mathrm{FC}$ с использованием более низких значений магнитного поля (100 Ое). В образцах с $x=0.2, x=0.25$ и $x=0.33$ были обнаружены максимумы при температурах 8,13 и $30 \mathrm{~K}$ соответственно, а также наличие гистерезиса ниже этих температур при измерениях в режимах ZFC и FC (рис. 5). Это может означать, что ниже критической температуры формируется состояние типа спинового или кластерного стекла. Отрицательные значения температуры Кюри-Вейсса, а также уменьшение магнитной восприимчивости в области низких температур при росте содержания магнитоактивных атомов позволяет предполагать существование в системе $\mathrm{Cr}_{x} \mathrm{VSe}_{2}$ антиферромагнитных взаимодействий. На это указывают также полевые зависимости намагниченности, приведенные на рис. 3 и демонстриру- ющие отсутствие насыщения вплоть до магнитного поля $6 \mathrm{kOe}$.

\section{4. Заключение}

Выполненные в настоящей работе исследования и полученные результаты показывают связь структурных преобразований при интеркалации диселенида ванадия атомами хрома с формируемыми физическими свойствами. Увеличение содержания хрома в $\mathrm{Cr}_{x} \mathrm{VSe}_{2}$ сопровождается упорядочением ионов хрома и вакансий в ВдВ-щелях и понижением симметрии элементарной ячейки от тригональной к моноклинной. Такое упорядочение и связанная с ним трансформация электронной структуры проявляется в изменении резистивного состояния, заключающегося в смене знака температурного коэффициента электросопротивления.

Определение эффективных магнитных моментов ионов хрома из данных по температурным зависимостям магнитной восприимчивости было произведено при учете возможного вклада исходной матрицы $\mathrm{VSe}_{2}$ Было показано, что величины $\mu_{\text {eff }} / \mathrm{Cr}$ для составов $\mathrm{c} x=0.05$ и $x=0.1$ практически соответствуют спиновому моменту ионов $\mathrm{Cr}^{3+}\left(3.87 \mu_{\mathrm{B}}\right)$, а при увеличении содержания хрома до $x=0.33$ уменьшается до $2.67 \mu_{\mathrm{B}}$. Сравнение концентрационных зависимостей $\mu_{\mathrm{eff}}$ и параметра $c_{0}$ элементарной ячейки позволяет сделать вывод о делокализации $3 d$-электронов хрома и наличии их гибридизации с электронными состояниями исходной матрицы. В области низких температур при содержании хрома $x \geq 0.2$ обнаружено существование спин-стекольных состояний с увеличивающейся температурой магнитного превращения до $30 \mathrm{~K}$ для $\mathrm{Cr}_{0.33} \mathrm{VSe}_{2}$. Уменьшение магнитной восприимчивости при росте содержания хрома, отрицательные значения температуры Кюри-Вейсса и отсутствие насыщения на полевых зависимостях намагниченности вплоть до $H=60 \mathrm{kOe}$ свидетельствуют об антиферромагнитном характере взаимодействия в температурной области спин-стекольного состояния. Таким образом, полученные в работе данные показывают, что в системе $\mathrm{Cr}_{x} \mathrm{VSe}_{2}$ при концентрациях хрома до $x=0.33$ не происходит установления дальнего магнитного порядка в отличие от таких хромосодержащих систем, как $\mathrm{Cr}_{x} \mathrm{TiSe}_{2}, \mathrm{Cr}_{x} \mathrm{TiTe}_{2}$ или $\mathrm{Cr}_{x} \mathrm{MoSe}_{2}$ [6,11,12]. Это подчеркивает важную роль, которую играет природа СДПМ при формировании физических свойств интеркалированных соединений.

\section{Финансирование работы}

Работа выполнена при финансовой поддержке Министерства науки и высшего образования Российской Федерации (проект № FEUZ-2020-0054).

\section{Конфликт интересов}

Авторы заявляют об отсутствии конфликта интересов. 


\section{Список литературы}

[1] W. Choi, N. Choudhary, J. Park, G.H. Ha, Y.H. Lii, D. Akinwande. Mater. Today 20, 3, 116 (2017). doi: 10.1016/j.mattod.2016.10.002

[2] Л.А. Чернозатонскй, А.А. Артюх УФН 188, 1, 3 (2018). doi: $10.3367 /$ UFNr.2017.02.038065

[3] A. Hayashi, Y. Ueda, K. Kosuge Mater. Res. Bull. 24, 7, 825 (1989). doi: 10.1016/0025-5408(89)90045-7

[4] A.S. Shkvarin, Y.M. Yarmoshenko, A.I. Merentsov, I. Pís, F. Bondino, E.G. Shkvarina, A.N. Titov. Inorganic Chem. 57, 9, 5544 (2018). doi: 10.1021/acs.inorgchem.8b00511

[5] Н.В. Селезнева, В.Г. Плещев, Н.В. Баранов. ФТТ 56, 12, 2293 (2014).

[6] N.V. Baranov, V.G. Pleshchev. Magnetic Properties of Layered Titanium Dichalcogenides Intercalated with 3d- and 4d-Metals. In: Handbook of Material Science Research / Eds Ch. René, E. Turcotte. Nova Science Publishers (2010). P. 295-322.

[7] В.Г. Плещев, А.Н. Титов, С.Г. Титова. ФТТ 45, 3, 409 (2003).

[8] C.S. Yadav, A.K. Rastogi. Solid State Commun. 150, 13-14, 648 (2010). doi: 10.1016/j.ssc.2009.12.026

[9] N.V. Selezneva, E.M. Sherokalova, V.G. Pleshchev, V.A. Kazantsev, N.V. Baranov. J. Phys.: Condens. Matter 28, 315401 (2016). doi: 10.1088/0953-8984/28/31/315401

[10] C.S. Yadav, A.K. Rastogi. J. Phys.: Condens. Matter 20, 46, 465219 (2008). doi: 10.1088/0953-8984/20/46/465219

[11] N.V. Baranov, V.G. Pleshchev, N.V. Selezneva, E.M. Sherokalova, A.V. Korolev, V.A. Kazantsev, A.V. Proshkin. J. Phys.: Condens. Matter 21, 50, 506002 (2009). doi: 10.1088/0953-8984/21/50/506002

[12] В.Г. Плещев, Н.В. Селезнева. ФТТ 61, 3, 472 (2019). doi: 10.21883/FTT.2019.03.47238.274

[13] Ф. Блатт. Физика электронной проводимости в твердых телах. Мир, М. (1971). 470 с.

[14] A.H. Reshak, S. Auluck. Physica B: Condens. Matter 349, 1-4, 310 (2004). doi: 10.1016/j.physb.2004.03.31314

Редактор Д.В. Жуманов 